\title{
Semi-inclusive back-to-back production of a hadron pair and a single hadron in $e^{+} e^{-}$annihilation
}

\author{
Hrayr H. Matevosyan, ${ }^{a}$ Aram Kotzinian ${ }^{b, c}$ and Anthony W. Thomas ${ }^{a}$ \\ ${ }^{a}$ ARC Centre of Excellence for Particle Physics at the Terascale and CSSM, Department of Physics, \\ The University of Adelaide, Adelaide SA 5005, Australia \\ ${ }^{b}$ Yerevan Physics Institute, \\ 2 Alikhanyan Brothers St., 375036 Yerevan, Armenia \\ ${ }^{c}$ INFN - Sezione di Torino, \\ 10125 Torino, Italy \\ E-mail: hrayr.matevosyan@adelaide.edu.au, aram.kotzinian@cern.ch, \\ anthony . thomas@adelaide.edu . au
}

ABSTRACT: Inclusive hadron production in $e^{+} e^{-}$annihilation has long been used to study both single hadron fragmentation functions $(\mathrm{FF})$ and dihadron fragmentation functions (DiFF). In particular, the polarized DiFFs can be accessed in electron-positron annihilation by measuring azimuthal correlations between two back-to-back pairs of hadrons in the center of mass system, where the relevant structure functions can be expressed as convolutions of two (polarized) DiFFs. Here we explore the advantages of measuring the inclusive back-to-back production of a single hadron on one side against a hadron pair on the opposite side of the detector in two jet events. The leading twist cross section for this process contains convolutions of the corresponding single hadron FFs on one side and the DiFFs for the hadron pair on the other side, which furnishes several interesting new opportunities. A measurement of the unpolarized cross section with a number of different types of observed hadrons will help in untangling the quark flavor dependence of the unpolarized DiFFs, when the results are analyzed together with the inclusive measurements of dihadron pairs, such as those recently performed by the BELLE collaboration. Even more interesting, with a polarized hyperon on one side we can study the quark spin-dependent DiFFs of an unpolarized hadron pair on the other side. This, in turn, will allow us to test the universality of the spin-dependent DiFFs entering the cross sections of electron-positron annihilation and semi-inclusive deep inelastic scattering processes.

KeYwords: Deep Inelastic Scattering (Phenomenology), QCD Phenomenology

ArXiv EPrint: 1808.00954 


\section{Contents}

1 Introduction 1

2 The cross section calculation 2

2.1 Kinematics 2

2.2 Cross section 4

3 The azimuthal asymmetries $\quad 10$

3.1 The asymmetry induced by the Collins effect for unpolarized $\Lambda \quad 10$

$\begin{array}{lll}3.2 & \text { The asymmetries for a longitudinally polarized } \Lambda & 11\end{array}$

3.3 The asymmetries for a transversely polarized $\Lambda \quad 12$

4 Treatment of the polarized $\Lambda^{0} \quad 14$

5 Conclusions $\quad 15$

\section{Introduction}

The study of the hadronization process, which is quantified by various fragmentation functions, has gained a great deal of attention in recent years [1]. This has been motivated by the ability of the new experiments to measure various azimuthal correlations in the deep inelastic scattering processes that involve polarized FFs and DiFFs. For example, the measurements of the quark transverse polarization dependent Collins FF and the so-called interference DiFF (IFF) in electron-positron annihilation in BELLE [2-5] and BaBar [6] experiments, have allowed the extraction of the quark transversity parton distribution function (PDF) [7-10] using semi-inclusive deep inelastic scattering (SIDIS) measurements with one and two detected final state hadrons by the HERMES [11, 12] and COMPASS [13, 14] collaborations. It is worth mentioning that the cross section for hadron production in $e^{+} e^{-}$ annihilation involves a summation over all intermediate quark-antiquark pairs, thus requiring additional input in order to access the quark flavor dependence of the FFs and DiFFs.

The key for such a combined analysis is the universality of the FFs and DiFFs entering the cross section of both electron-positron annihilation and SIDIS processes, which was proven explicitly for the transverse-momentum dependent FFs [15, 16], while similar arguments should apply in the case of DiFFs [17]. This is in contrast to the prediction of the process dependence of the naive-time-reversal-odd (T-odd) Sivers PDF, which is predicted to change sign between SIDIS and Drell-Yan annihilation [18]. An experimental test of such universality for the naive-time-reversal-odd FFs has been proposed in ref. [19], by exploring the so-called "polarizing" FF $D_{1 T}^{\perp}$ of a spin $1 / 2$ hyperon. The "polarizing" FF describes the correlation of the transverse polarization of a produced hadron with its 
own transverse momentum in the quark fragmentation process and is a chiral-even function. Thus, in SIDIS with a final state hyperon detected, it couples to the well-determined unpolarized PDF, while in $e^{+} e^{-}$annihilation with back-to-back hyperon and unpolarized hadron production, it couples to the corresponding unpolarized FF.

Recently, we proposed two new measurements [20] of the quark helicity dependent DiFF $G_{1}^{\perp}$, both in SIDIS with two final state detected hadrons and two back-to-back hadron pair production in $e^{+} e^{-}$annihilation. The chiral-even nature of $G_{1}^{\perp}$ entails that in SIDIS it couples to the well-determined quark unpolarized and helicity PDFs [21]. In $e^{+} e^{-}$annihilation though, the $G_{1}^{\perp}$ from one pair couples to that for the second pair on the other side [22, 23], making it not possible (or at least very hard) to determine the sign of this function. Thus, such measurements allow one to test only for the agreement between the magnitudes of $G_{1}^{\perp}$ extracted from the two measurements.

In this work we propose a new measurement in $e^{+} e^{-}$annihilation, exploiting the production of a single inclusive hadron back-to-back to a hadron pair, where the relevant cross section should involve convolutions of FFs for the single hadron in one jet and the DiFFs for the hadron pair produced in the opposite jet. The purpose of such a measurement is two-fold, and will leverage our knowledge of the single hadron FFs. First, the absolute cross section measurements will provide a wider basis for extracting the quark flavor dependence of the DiFFs, especially when analyzed together with the inclusive hadron pair measurements in the same jet [5]. Secondly, by studying various azimuthal asymmetries we can better determine the polarized DiFFs, and also access their sign.

This paper is organized in the following way. In the next section we detail the derivation of the cross section for the proposed new process. In section 3, we explore the relevant unpolarized measurements and the azimuthal asymmetries for accessing the DiFFs. In section 4 , we examine the particular case where one detects a polarized $\Lambda^{0}$ hyperon. Finally, we present the summary of our findings and conclusions in section 5 .

\section{The cross section calculation}

In this section we detail the derivation of the leading twist cross section for the process $e^{+} e^{-} \rightarrow h_{1} h_{2}+\Lambda+X$, where the electron and positron with momenta $l$ and $l^{\prime}$ annihilate into an intermediate virtual photon with momentum $q=l+l^{\prime}$. In the final state, we detect a hadron $\Lambda$ with momentum $P_{\Lambda}$, produced back-to-back to the unpolarized hadron pair $h_{1}, h_{2}$ of momenta $P_{1}$ and $P_{2}$, as illustrated in figure 1 . Here $\Lambda$ denotes either an unpolarized hadron ( for example $\pi, K$, etc ) hadron, or a spin $1 / 2$ baryon withe a polarization vector $\boldsymbol{S}_{\Lambda}$. We restrict our consideration to the case where the center-of-mass energy of the electron-positron pair is far below the mass of the $Z$ boson. We use the conventional framework for the inclusive hadron production in $e^{+} e^{-}$annihilation [22-25]. In the next subsection we first describe the kinematics of the process and then detail the calculation of the cross section in the following subsection.

\subsection{Kinematics}

For our calculations we choose the $e^{+} e^{-}$center-of-mass coordinate system, where the $\hat{z}$ axis is chosen to point opposite to the $\Lambda$ 's 3 -momentum $\boldsymbol{P}_{\Lambda}$. We denote the components 


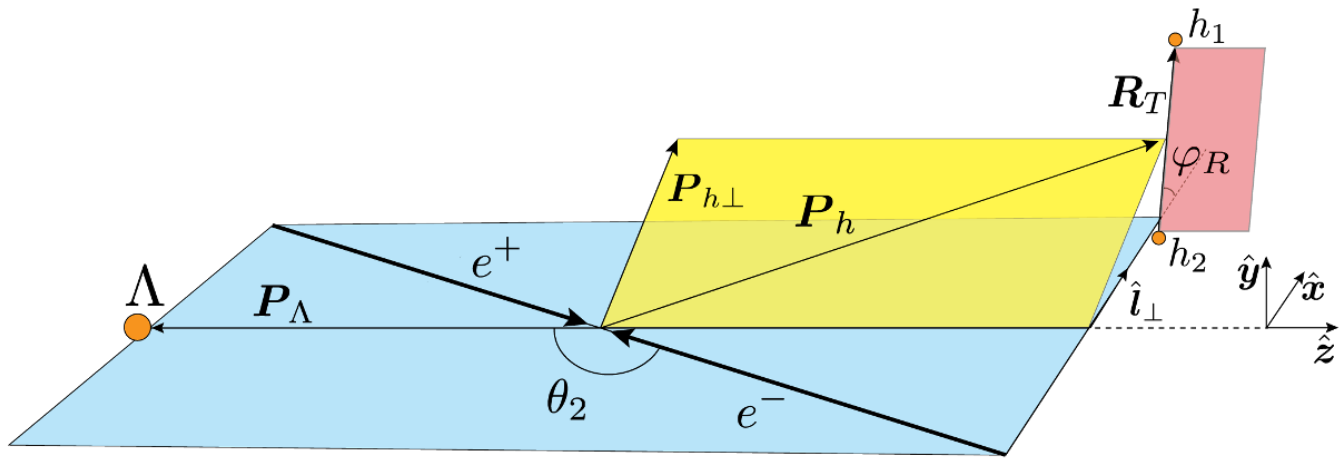

Figure 1. The kinematics of $e^{+} e^{-} \rightarrow h_{1} h_{2}+\Lambda+X$ process.

of 3 -vectors transverse to $\hat{z}$ axis with subscript $\perp$. The $\hat{x}$ axis is then taken along the transverse component $\boldsymbol{l}_{\perp}$ of the electron momentum. Together, the $\hat{z}$ and $\hat{x}$ axes span the lepton plane. In dihadron studies it is useful to replace $P_{1}, P_{2}$ by the total and the relative hadron momenta, defined as

$$
\begin{aligned}
P_{h} & =P_{1}+P_{2}, \\
R & =\frac{1}{2}\left(P_{1}-P_{2}\right),
\end{aligned}
$$

and the invariant mass of the pair is defined by $P_{h}^{2}=M_{h}^{2}$.

We consider the kinematic regime where the virtuality of the time like momentum of the virtual photon is much larger than the typical hadronic mass scales, thus defining $Q^{2} \equiv q^{2}$ we can ignore any contributions of order $1 / Q$. At the QED and QCD leading order approximation, the virtual photon produces a quark and an antiquark pair $e^{+} e^{-} \rightarrow \gamma^{*} \rightarrow$ $q+\bar{q}$, which then hadronize and produce two back-to-back jets. Further, we assume that we are in the "leading hadron approximation", where a significant fraction of the energy in each jet is carried by the observed hadrons, that is $P_{h} \cdot P_{\Lambda} \sim Q^{2}$. We can use these two large momenta to define light-like directions to conveniently decompose the hadronic tensor, as done earlier for other reactions [22, 24]. For this, we choose a coordinate system where the transverse components of the light-cone momenta ${ }^{1}$ of $P_{h}, P_{\Lambda}$ vanish, $\boldsymbol{P}_{h T}=0$ and $\boldsymbol{P}_{\Lambda T}=0$. Introducing two light-like vectors $n_{+}$and $n_{-}$, such that $n_{+}^{2}=n_{-}^{2}=0$ and $n_{+} n_{-}=1$, we can decompose the relevant large momenta as

$$
\begin{aligned}
P_{h}^{\mu} & =\frac{M_{h}^{2}}{z_{h} Q \sqrt{2}} n_{-}^{\mu}+\frac{z_{h} Q}{\sqrt{2}} n_{+}^{\mu} \approx \frac{z_{h} Q}{\sqrt{2}} n_{+}^{\mu}, \\
P_{\Lambda}^{\mu} & =\frac{z_{\Lambda} Q}{\sqrt{2}} n_{-}^{\mu}+\frac{M_{\Lambda}^{2}}{z_{\Lambda} Q \sqrt{2}} n_{+}^{\mu} \approx \frac{z_{\Lambda} Q}{\sqrt{2}} n_{-}^{\mu}, \\
q^{\mu} & =\frac{Q}{\sqrt{2}} n_{-}^{\mu}+\frac{Q}{\sqrt{2}} n_{+}^{\mu}+q_{T}^{\mu},
\end{aligned}
$$

\footnotetext{
${ }^{1}$ The light-cone components of a 4 -vector $a$ are defined as $a=\left(a^{+}, a^{-}, \boldsymbol{a}_{T}\right)$, where $a^{ \pm}=\frac{1}{\sqrt{2}}\left(a^{0} \pm a^{3}\right)$.
} 
where $M_{\Lambda}$ is the mass of $\Lambda$, and the momentum fractions are defined as

$$
\begin{aligned}
& z_{h}=\frac{2 P_{h} \cdot q}{Q^{2}} \approx \frac{P_{h}^{+}}{q^{+}} \equiv z, \\
& z_{\Lambda}=\frac{2 P_{\Lambda} \cdot q}{Q^{2}} \approx \frac{P_{\Lambda}^{-}}{q^{-}} \equiv \bar{z}
\end{aligned}
$$

and

$$
-q_{T}^{2}=Q_{T}^{2} \ll Q^{2}
$$

In this system, for any 4 -vector, we can obtain the components orthogonal to $n_{ \pm}$using

$$
\begin{aligned}
g_{T}^{\mu \nu} & =g^{\mu \nu}-n_{+}^{\mu} n_{-}^{\nu}-n_{+}^{\nu} n_{-}^{\mu}, \\
\epsilon_{T}^{\mu \nu} & =\epsilon^{\mu \nu \rho \sigma} n_{+\rho} n_{-\sigma},
\end{aligned}
$$

where $g^{\mu \nu}$ is the metric tensor and we use the convention $\epsilon^{0123}=+1$.

The leptonic tensor, on the other hand, is usually evaluated in the coordinate system depicted in figure 1 , where $\boldsymbol{P}_{\Lambda}=\boldsymbol{q}_{\perp}=0$. In this system, we can again define normalized time-like and space like vectors along the dominant momenta

$$
\begin{aligned}
\hat{t} & =\frac{q}{Q}, \\
\hat{v} & =2 \frac{P_{\Lambda}}{\bar{z} Q}-\hat{t} .
\end{aligned}
$$

The projections of the 4 -vectors onto the two orthogonal $\perp$ directions can be obtained using the tensors

$$
\begin{aligned}
& g_{\perp}^{\mu \nu}=g^{\mu \nu}-\hat{t}^{\mu} \hat{t}^{\nu}+\hat{v}^{\nu} \hat{v}^{\mu}, \\
& \epsilon_{\perp}^{\mu \nu}=-\epsilon^{\mu \nu \rho \sigma} \hat{t}_{\rho} \hat{v}_{\sigma} .
\end{aligned}
$$

Interestingly, the two perpendicular projection tensors differ only by a factor of order $Q_{T} / Q$, since

$$
g_{\perp}^{\mu \nu}=g_{T}^{\mu \nu}-\frac{n_{+}^{\mu} q_{T}^{\nu}+n_{+}^{\nu} q_{T}^{\mu}}{Q} .
$$

Thus we can neglect the differences between the $T$ and $\perp$ components of the vectors in this work.

\subsection{Cross section}

The cross section for this process is given by the convolution of the leptonic and hadronic tensors

$$
\frac{2 P_{1}^{0} 2 P_{2}^{0} 2 P_{\Lambda}^{0} d \sigma}{d^{3} \boldsymbol{P}_{1} d^{3} \boldsymbol{P}_{2} d^{3} \boldsymbol{P}_{\Lambda}}=\frac{\alpha_{e m}^{2}}{Q^{6}} L_{\mu \nu} W_{(3 h)}^{\mu \nu},
$$


where $L_{\mu \nu}$ is the leptonic tensor, $W_{(3 h)}^{\mu \nu}$ is the hadronic tensor, and $\alpha_{e m}$ is the fine structure constant. The leptonic tensor can be evaluated using standard methods (e.g. [24]), yielding

$$
\begin{aligned}
L_{\mu \nu}=Q^{2}\left[-2 A(y) g_{\perp}^{\mu \nu}+4 B(y) \hat{v}^{\mu} \hat{v}^{\nu}\right. & -4 B(y)\left(\hat{l}_{\perp}^{\mu} \hat{l}_{\perp}^{\nu}+\frac{1}{2} g_{\perp}^{\mu \nu}\right) \\
& \left.-2 C(y) B^{1 / 2}(y)\left(\hat{v}^{\mu} \hat{l}_{\perp}^{\nu}+\hat{v}^{\nu} \hat{l}_{\perp}^{\mu}\right)\right],
\end{aligned}
$$

where the normalized perpendicular part of the leptons's 4-momentum is defined as

$$
\hat{l}_{\perp}^{\mu}=\frac{l_{\perp}^{\mu}}{\left|\boldsymbol{l}_{\perp}\right|}
$$

The usual coefficient functions are given by

$$
\begin{aligned}
& A(y)=\frac{1}{2}-y+y^{2}, \\
& B(y)=y(1-y), \\
& C(y)=1-2 y,
\end{aligned}
$$

where the lepton momentum fractions are defined as

$$
y=\frac{P_{h} \cdot l}{P_{h} \cdot q} \approx \frac{l^{-}}{q^{-}}=\frac{1+\cos \theta_{2}}{2} .
$$

Here $\theta_{2}$ is the angle between the 3 -momentum of the electron $l$ and the $\hat{z}$ axis in the center-of-mass frame,

The phase space factor can be then written in the conventional notation

$$
d^{9} V \equiv \frac{d^{3} \boldsymbol{P}_{1} d^{3} \boldsymbol{P}_{2} d^{3} \boldsymbol{P}_{\Lambda}}{2 P_{1}^{0} 2 P_{2}^{0} 2 P_{\Lambda}^{0}}=\frac{d^{2} \boldsymbol{P}_{h \perp} d z d \varphi_{R} d M_{h}^{2} d \xi}{8 z} \frac{Q^{2} \bar{z} d \bar{z} d \Omega_{\Lambda}}{8},
$$

where we used $\boldsymbol{P}_{\Lambda \perp}=0$ and the leading order relations in the hard scale $Q$

$$
\begin{aligned}
& P_{h}^{-} \ll P_{h}^{+}, \rightarrow E_{h} \sim \frac{1}{\sqrt{2}} P_{h}^{+}, \\
& R^{-} \ll R^{+}, \rightarrow E_{R} \sim \frac{1}{\sqrt{2}} R^{+}, \\
& \frac{R^{+}}{P_{h}^{+}}=\xi-1 / 2 \sim \frac{E_{R}}{E_{h}},
\end{aligned}
$$

and we replaced $\left|\boldsymbol{R}_{T}\right|$ with the invariant mass of the hadron pair $M_{h}$ using the relation

$$
\boldsymbol{R}_{T}^{2}=\xi(1-\xi) M_{h}^{2}-M_{1}^{2}(1-\xi)-M_{2}^{2} \xi .
$$

The spherical angle of the $\Lambda$ hadron can be expressed in terms of $y$ and the azimuthal angle of $\boldsymbol{l}_{\perp}$ as

$$
d \Omega_{\Lambda}=2 d y d \varphi_{L}
$$


yielding

$$
\begin{aligned}
d^{9} V & =\frac{Q^{2}}{32} \frac{\bar{z}}{z} d^{2} \boldsymbol{P}_{h \perp} d z d \varphi_{R} d M_{h}^{2} d \xi d \bar{z} d y d \varphi_{L} \\
& =z \bar{z} \frac{Q^{2}}{32} d^{2} \boldsymbol{q}_{T} d z d \varphi_{R} d M_{h}^{2} d \xi d \bar{z} d y d \varphi_{L}
\end{aligned}
$$

where in the last expression we substituted $\boldsymbol{P}_{h \perp}$ with the transverse component of the intermediate virtual photon's 3-momentum in the light-cone frame $\boldsymbol{q}_{T}=-\boldsymbol{P}_{h \perp} / z$.

The hadronic tensor is defined as

$$
\begin{aligned}
W_{(3 h)}^{\mu \nu}\left(q ; P_{h}, R, P_{\Lambda}\right)=\frac{1}{(2 \pi)^{7}} \sum_{X} \int & \frac{d^{3} \boldsymbol{P}_{X}}{(2 \pi)^{3} 2 P_{X}^{0}}(2 \pi)^{4} \delta\left(q-P_{X}-P_{h}-P_{\Lambda}\right) \\
& \times\left\langle 0\left|J^{\mu}(0)\right| P_{X} ; P_{h}, R, P_{\Lambda}\right\rangle\left\langle P_{X} ; P_{h}, R, P_{\Lambda}\left|J^{\nu}(0)\right| 0\right\rangle
\end{aligned}
$$

Using the parton picture, we can decompose the hadronic tensor in terms of the quarkquark correlators $\Delta$ and $\bar{\Delta}$ for the production of a hadron pair and a hyperon in the fragmentation of the quark and the antiquark with momenta $k$ and $\bar{k}$, respectively

$$
W_{(3 h)}^{\mu \nu} \approx \frac{3}{(2 \pi)^{3}} \sum_{a} e_{a}^{2} \int d^{4} k d^{4} \bar{k} \delta^{4}(q-k-\bar{k}) \operatorname{Tr}\left[\bar{\Delta}\left(\bar{k}, P_{\Lambda}\right) \gamma^{\mu} \Delta\left(k, P_{h}, R\right) \gamma^{\mu}\right],
$$

where $a$ and $e_{a}$ denote the flavor and the fractional electric charge of the fragmenting quark, while the pre factor is the number of quark colors $N_{c}=3$. We can then integrate the $k^{+}$ and $\bar{k}^{-}$components using the $\delta$ function and rewrite the hadronic tensor as a convolution of the conventional integrated quark-quark correlators

$$
\begin{aligned}
& W_{(3 h)}^{\mu \nu} \approx \frac{3(32 z)(4 \bar{z})}{(2 \pi)^{3}} \sum_{a} e_{a}^{2} \int d^{2} \boldsymbol{k}_{T} d^{2} \overline{\boldsymbol{k}}_{T} \delta^{2}\left(\boldsymbol{q}_{T}-\boldsymbol{k}_{T}-\overline{\boldsymbol{k}}_{T}\right) \\
& \times \operatorname{Tr}\left[\bar{\Delta}\left(\bar{z}, \overline{\boldsymbol{k}}_{T}\right)_{\bar{k}}=P_{\Lambda}^{-} / \bar{z} \gamma^{\mu} \Delta\left(z, \xi, \boldsymbol{k}_{T}, \boldsymbol{R}_{T}\right)_{k^{+}=P_{h}^{+} / z} \gamma^{\mu}\right],
\end{aligned}
$$

where the light-cone momentum fractions for the dihadron correlators are defined in terms of those for the individual hadrons,

$$
\begin{aligned}
& z=z_{1}+z_{2}, \\
& \xi=\frac{z_{1}}{z}=1-\frac{z_{2}}{z},
\end{aligned}
$$

where $z_{i}=P_{i}^{+} / k^{+}$are the light-cone momentum fractions of each hadron in the pair.

The two-hadron fragmentation of a quark is described by a quark-quark correlator $[22$, $26-28]$

$$
\Delta_{i j}\left(k ; P_{h}, R\right)=\sum_{X} \int d^{4} \zeta e^{i k \cdot \zeta}\left\langle 0\left|\psi_{i}(\zeta)\right| P_{h} R, X\right\rangle\left\langle P_{h} R, X\left|\bar{\psi}_{j}(0)\right| 0\right\rangle,
$$

which, for the case of an unpolarized hadron pair and at the leading twist approximation, is parametrized via four DiFFs

$$
\begin{aligned}
& \left.\frac{1}{32 z} \int d k^{-} \Delta\left(k, P_{h}, R\right)\right|_{k^{+}=P_{h}^{+} / z} \equiv \Delta\left(z, \xi, \boldsymbol{k}_{T}, \boldsymbol{R}_{T}\right) \\
& \quad=\frac{1}{4 \pi} \frac{1}{4}\left\{D_{1} \not h_{+}-G_{1}^{\perp} \frac{\epsilon_{\mu \nu \rho \sigma} \gamma^{\mu} n_{+}^{\nu} k_{T}^{\rho} R_{T}^{\sigma}}{M_{h}^{2}} \gamma_{5}+H_{1}^{\varangle} \frac{\sigma_{\mu \nu} R_{T}^{\mu} n_{+}^{\nu}}{M_{h}}+H_{1}^{\perp} \frac{\sigma_{\mu \nu} k_{T}^{\mu} n_{+}^{\nu}}{M_{h}}\right\} .
\end{aligned}
$$


Here $D_{1}$ is the unpolarized DiFF, while the helicity-dependent DiFF, $G_{1}^{\perp}$, describes the correlation of the fragmenting quark's longitudinal polarization with the vector product of the transverse momenta of the two hadrons. The two remaining DiFFs, $H_{1}^{\varangle}$ (IFF) and $H_{1}^{\perp}$, describe the correlations between the quark transverse polarization with the relative and total transverse momenta of the pair, respectively. The DiFFs here are defined in a frame where their total momentum 3-momentum is along the $\hat{z}$ axis, and we can choose as two transverse vectors $\boldsymbol{R}_{T}$ and $\boldsymbol{k}_{T}$, which are the transverse components of $R$ and $k$ in this system. We can relate $\boldsymbol{k}_{T}=-\boldsymbol{P}_{\perp} / z$ to the transverse component of $P$ in a frame where $k$ has no transverse component. In general, DiFFs depend on $z, \xi, \boldsymbol{k}_{T}, \boldsymbol{R}_{T}$. It is worth noting, that only $\boldsymbol{k}_{T} \cdot \boldsymbol{R}_{T}$ correlations are possible for our scalar functions and so the azimuthal dependence of the DiFFs can be expanded in a Fourier cosine series

$$
D_{1}\left(z, \xi, \boldsymbol{k}_{T}^{2}, \boldsymbol{R}_{T}^{2}, \cos \left(\varphi_{K R}\right)\right)=\frac{1}{\pi} \sum_{n=0}^{\infty} \frac{\cos \left(n \cdot \varphi_{K R}\right)}{1+\delta^{0, n}} D_{1}^{[n]}\left(z, \xi,\left|\boldsymbol{k}_{T}\right|,\left|\boldsymbol{R}_{T}\right|\right),
$$

where $\varphi_{K R} \equiv \varphi_{k}-\varphi_{R}$ is the relative azimuthal angle between $\boldsymbol{k}_{T}$ and $\boldsymbol{R}_{T}$.

For single polarized hadron production the quark-quark correlator now depends on the momentum of the fragmenting quark $k$, and the momentum $P_{\Lambda}$ and polarization $\boldsymbol{S}_{\Lambda}$ of the produced hadron. Once again we can integrate over the large component of the light-cone momentum

$$
\frac{1}{4 z} \int d k^{+} \Delta\left(k, P_{\Lambda}, \boldsymbol{S}_{\Lambda}\right)_{k^{-}=P_{\Lambda}^{-} / z} \equiv \Delta\left(z, \boldsymbol{k}_{T}, \boldsymbol{S}_{\Lambda}\right),
$$

where $\boldsymbol{k}_{T}$ is the transverse component of the fragmenting quark's momentum in a system with the $\hat{z}$ axis pointed along the 3 -momentum $\boldsymbol{P}_{\Lambda}$. Following the notation in ref. [29], we can expand the correlator in terms of the relevant leading-twist transverse momentum dependent (TMD) FFs

$$
\begin{aligned}
\Delta\left(z, \boldsymbol{k}_{T}, \boldsymbol{S}_{\Lambda}\right)=\frac{1}{4}\{ & D_{1} \not \hbar_{+}+D_{1 T}^{\perp} \frac{\epsilon_{\mu \nu \rho \sigma} \gamma^{\mu} n_{+}^{\nu} k_{T}^{\rho} S_{T}^{\sigma}}{M_{h}} \\
& -\left(\lambda_{\Lambda} G_{1 L}+G_{1 T} \frac{\boldsymbol{k}_{T} \cdot \boldsymbol{S}_{T}}{M_{h}}\right) h_{+} \gamma_{5}-H_{1 T} i \sigma_{\mu \nu} S_{T}^{\mu} n_{+}^{\nu} \gamma_{5} \\
& \left.-\left(\lambda_{\Lambda} H_{1 L}^{\perp}+H_{1 T}^{\perp} \frac{\boldsymbol{k}_{T} \cdot \boldsymbol{S}_{T}}{M_{h}}\right) i \sigma_{\mu \nu} k_{T}^{\mu} n_{+}^{\nu} \gamma_{5}+H_{1}^{\perp} \frac{\sigma_{\mu \nu} k_{T}^{\mu} n_{+}^{\nu}}{M_{h}}\right\},
\end{aligned}
$$

where $\lambda_{\Lambda}$ and $\boldsymbol{S}_{T}$ are the helicity and the transverse polarization vectors of the produced $\Lambda$ hadron. The FFs are functions of $z$ and $\boldsymbol{P}_{\Lambda \perp}^{2}$. Here $\boldsymbol{P}_{\Lambda \perp}=-z \boldsymbol{k}_{T}$ is the transverse momentum of the produced hadron in a system where the 3 -momentum of the quark $\boldsymbol{k}$ has no transverse component.

Finally, we can calculate the cross section

$$
\begin{aligned}
& \frac{d \sigma\left(e^{+} e^{-} \rightarrow\left(h_{1} h_{2}\right)+\Lambda+X\right)}{d^{2} \boldsymbol{q}_{T} d z d \varphi_{R} d M_{h}^{2} d \xi d \bar{z} d y}=\frac{3 \alpha_{e m}^{2}}{(2 \pi)^{2} Q^{2}} z^{2} \bar{z}^{2} \sum_{a} e_{a}^{2} \\
& \times\left\{A(y) \mathcal{F}\left[D_{1}^{a \rightarrow h_{1} h_{2}} D_{1}^{\bar{a} \rightarrow \Lambda}\right]\right. \\
& -S_{T} A(y) \mathcal{F}\left[\frac{\bar{k}_{T}}{M_{\Lambda}} \sin \left(\varphi_{\bar{k}}-\varphi_{S}\right) D_{1}^{a \rightarrow h_{1} h_{2}} D_{1 T}^{\perp, \bar{a} \rightarrow \Lambda}\right]
\end{aligned}
$$




$$
\begin{aligned}
&+\lambda_{\Lambda} A(y) \mathcal{F}[\left.\frac{k_{T} R_{T}}{M_{h}^{2}} \sin \left(\varphi_{k}-\varphi_{R}\right) G_{1}^{\perp, a \rightarrow h_{1} h_{2}} G_{1 L}^{\bar{a} \rightarrow \Lambda}\right] \\
&+S_{T} A(y) \mathcal{F}\left[\frac{k_{T} R_{T}}{M_{h}^{2}} \sin \left(\varphi_{k}-\varphi_{R}\right) \frac{\bar{k}_{T}}{M_{\Lambda}} \cos \left(\varphi_{\bar{k}}-\varphi_{S}\right) G_{1}^{\perp, a \rightarrow h_{1} h_{2}} G_{1 T}^{\bar{a} \rightarrow \Lambda}\right] \\
&+S_{T} B(y) \mathcal{F}\left[\left(\frac{k_{T}}{M_{h}} \sin \left(\varphi_{k}+\varphi_{S}\right) H_{1}^{\perp, a \rightarrow h_{1} h_{2}}\right.\right. \\
&\left.\left.+\frac{R_{T}}{M_{h}} \sin \left(\varphi_{R}+\varphi_{S}\right) H_{1}^{\varangle, a \rightarrow h_{1} h_{2}}\right) H_{1 T}^{\bar{a} \rightarrow \Lambda}\right] \\
&+\lambda_{\Lambda} B(y) \mathcal{F}\left[\left(\frac{k_{T}}{M_{h}} \sin \left(\varphi_{k}+\varphi_{\bar{k}}\right) H_{1}^{\perp, a \rightarrow h_{1} h_{2}}\right.\right. \\
&\left.\left.\quad+\frac{R_{T}}{M_{h}} \sin \left(\varphi_{R}+\varphi_{\bar{k}}\right) H_{1}^{\varangle, a \rightarrow h_{1} h_{2}}\right) \frac{\bar{k}_{T}}{M_{\Lambda}} H_{1 L}^{\perp, \bar{a} \rightarrow \Lambda}\right] \\
&+S_{T} B(y) \mathcal{F}\left[\left(\frac{k_{T}}{M_{h}} \sin \left(\varphi_{k}+\varphi_{\bar{k}}\right) H_{1}^{\perp, a \rightarrow h_{1} h_{2}}\right.\right. \\
&\left.\left.\quad+\frac{R_{T}}{M_{h}} \sin \left(\varphi_{R}+\varphi_{\bar{k}}\right) H_{1}^{\varangle, a \rightarrow h_{1} h_{2}}\right) \frac{\bar{k}_{T}^{2}}{M_{\Lambda}^{2}} \cos \left(\varphi_{\bar{k}}-\varphi_{S}\right) H_{1 T}^{\perp, \bar{a} \rightarrow \Lambda}\right] \\
&+B(y) \mathcal{F}\left[\left(\frac{k_{T}}{M_{h}} \cos \left(\varphi_{k}+\varphi_{\bar{k}}\right) H_{1}^{\perp, a \rightarrow h_{1} h_{2}}\right.\right. \\
&\left.\left.\left.\quad+\frac{R_{T}}{M_{h}} \cos \left(\varphi_{R}+\varphi_{\bar{k}}\right) H_{1}^{\varangle, a \rightarrow h_{1} h_{2}}\right) \frac{\bar{k}_{T}}{M_{\Lambda}} H_{1}^{\perp, \bar{a} \rightarrow \Lambda}\right]\right\},
\end{aligned}
$$

where $\varphi_{S}$ is the azimuthal angle of $\boldsymbol{S}_{T}$ and we have integrated over the trivial dependence on the azimuthal angle of the lepton plane $\varphi_{L}$. The transverse momentum convolution, $\mathcal{F}$, is defined as

$$
\begin{aligned}
\mathcal{F} & {\left[w D^{a} \bar{D}^{\bar{a}}\right] } \\
& =\int d^{2} \boldsymbol{k}_{T} d^{2} \overline{\boldsymbol{k}}_{T} \delta^{2}\left(\boldsymbol{k}_{T}+\overline{\boldsymbol{k}}_{T}-\boldsymbol{q}_{T}\right) w\left(\boldsymbol{k}_{T}, \overline{\boldsymbol{k}}_{T}, \boldsymbol{R}_{T}, \overline{\boldsymbol{R}}_{T}\right) D^{a}\left(z, \xi, \boldsymbol{k}_{T}^{2}, \boldsymbol{R}_{T}^{2}, \boldsymbol{k}_{T} \cdot \boldsymbol{R}_{T}\right) D^{\bar{a}}\left(\bar{z}, \overline{\boldsymbol{k}}_{T}^{2}\right) .
\end{aligned}
$$

Integrating the cross section over $\boldsymbol{q}_{T}, \varphi_{R}$ and $\xi$ yields the unpolarized "collinear" cross section

$$
\frac{d \sigma\left(e^{+} e^{-} \rightarrow\left(h_{1} h_{2}\right)+\Lambda+X\right)}{d z d M_{h}^{2} d \bar{z} d y}=\frac{3 \alpha_{e m}^{2}}{(2 \pi)^{2} Q^{2}} A(y) \sum_{a} e_{a}^{2} D_{1}^{a \rightarrow h_{1} h_{2}}\left(z, M_{h}^{2}\right) \bar{D}_{1}^{\bar{a} \rightarrow \Lambda}(\bar{z}),
$$

where

$$
\begin{aligned}
D_{1}^{a}\left(z, M_{h}^{2}\right) & =z^{2} \int d^{2} \boldsymbol{k}_{T} \int d \xi \int d \varphi_{R} D_{1}^{a}\left(z, \xi, \boldsymbol{k}_{T}^{2}, \boldsymbol{R}_{T}^{2}, \boldsymbol{k}_{T} \cdot \boldsymbol{R}_{T}\right) \\
& =z^{2} \int d^{2} \boldsymbol{k}_{T} \int d \xi D_{1}^{a,[0]}\left(z, \xi,\left|\boldsymbol{k}_{T}\right|,\left|\boldsymbol{R}_{T}\right|\right)
\end{aligned}
$$

and

$$
D_{1}^{\bar{a}}(z)=\bar{z}^{2} \int d^{2} \overline{\boldsymbol{k}}_{T} D_{1}^{\bar{a}}\left(\bar{z}, \overline{\boldsymbol{k}}_{T}^{2}\right)
$$


Further integrating the result in eq. (2.43) over $M_{h}^{2}$ we recover the same expression as for two back-to-back hadron production in eq. (77) of ref. [24]. This is natural, as the integrated DIFF

$$
D_{1}^{a \rightarrow h_{1} h_{2}}(z) \equiv \int d M_{h}^{2} D_{1}^{a \rightarrow h_{1} h_{2}}\left(z, M_{h}^{2}\right),
$$

can be thought of describing the collinear fragmentation into a "parent" particle that carries the total light-cone momentum fraction $z$ of the pair $h_{1} h_{2}$.

The cross sections of the measurements of a hadron pair in the same hemisphere, such as those recently performed by the BELLE collaboration [5, 30], involve a sum over all the fragmenting quark flavors. Thus, leveraging the relatively well-determined single hadron FFs in a combined analysis of the single hadron pair cross section with that in eq. (2.43) would enable the flavor separation of the unpolarized DiFFs.

For example, for DiFF into $\pi^{+} \pi^{-}$pairs, the isospin and charge symmetries entail

$$
\begin{aligned}
& D_{1}^{u \rightarrow \pi^{+} \pi^{-}}=D_{1}^{\bar{u} \rightarrow \pi^{+} \pi^{-}} \approx D_{1}^{d \rightarrow \pi^{+} \pi^{-}}=D_{1}^{\bar{d} \rightarrow \pi^{+} \pi^{-}}, \\
& D_{1}^{s \rightarrow \pi^{+} \pi^{-}}=D_{1}^{\bar{s} \rightarrow \pi^{+} \pi^{-}} .
\end{aligned}
$$

For the one pair inclusive production, omitting the contributions of all the heavier flavors, we can approximate

$$
d \sigma\left(e^{+} e^{-} \rightarrow\left(h_{1} h_{2}\right)+X\right) \sim \sum_{q} e_{q}^{2} D_{1}^{q \rightarrow \pi^{+} \pi^{-}} \approx \frac{5}{9} D_{1}^{u \rightarrow \pi^{+} \pi^{-}}(z)+\frac{1}{9} D_{1}^{s \rightarrow \pi^{+} \pi^{-}}(z) .
$$

Hence the separation of the light and strange quark DiFF contributions is not possible without additional input. For the associated hadron production in the opposite hemisphere, the cross section can be approximated as

$$
d \sigma\left(e^{+} e^{-} \rightarrow\left(h_{1} h_{2}\right)+\pi^{+}+X\right) \sim \frac{5}{9} D_{1}^{u \rightarrow \pi^{+} \pi^{-}}(z) D_{1}^{u^{+} \rightarrow \pi^{+}}(\bar{z})+\frac{1}{9} D_{1}^{s \rightarrow \pi^{+} \pi^{-}}(z) D_{1}^{s^{+} \rightarrow \pi^{+}}(\bar{z}),
$$

where

$$
D_{1}^{q^{+} \rightarrow h}(\bar{z}) \equiv D_{1}^{q \rightarrow h}(\bar{z})+D_{1}^{\bar{q} \rightarrow h}(\bar{z}) .
$$

The unpolarized FFs to charged pions have been phenomenologically extracted from experimental measurement by a number of different groups [31-35]. Thus the measurement of the cross section in eq. (2.50) would allow us to extract the flavor dependence of the DiFF $D_{1}^{q \rightarrow \pi^{+} \pi^{-}}$. Similar arguments can be used to explore other hadron channels and combinations. For example, choosing a $\pi^{+} K^{+}$pair on one side and a $K^{-}$hadron on the other side would significantly enhance the "double favored" channel $\left(\bar{s} \rightarrow \pi^{+} K^{+}, s \rightarrow K^{-}\right)$ for moderate to large values of $z_{1}, z_{2}$, and $\bar{z}$, as can also be seen in hadronization models [3639]. The contributions of all the other quark flavors would include at least one "unfavored" fragmentation, which will be suppressed for our chosen region of light-cone momentum fractions. Thus, such a measurement would allow us to extract a DiFF for a specific quark flavor, $\bar{s} \rightarrow \pi^{+} K^{+}$. In principle, a global fit, using all possible measurements of the single and dihadron fragmentations would yield the best estimates of these functions, with the most realistic uncertainties. 


\section{The azimuthal asymmetries}

In this section we will discuss several asymmetries that give access to various combinations of polarization-dependent FFs and DiFFs, that can be measured at BELLE and the upcoming BELLE II experiments, similar to the measurements of the asymmetries for backto-back hadrons $[28,40]$ and back-to-back hadron pairs $[22,23]$.

We define the integral of the cross section in eq. (2.41), weighted with an arbitrary function $\mathcal{I}$, as

$$
\langle\mathcal{I}\rangle \equiv \int d \xi \int d \varphi_{R} \int d^{2} \boldsymbol{q}_{T} \mathcal{I} \frac{d \sigma\left(e^{+} e^{-} \rightarrow\left(h_{1} h_{2}\right)+\Lambda+X\right)}{d^{2} \boldsymbol{q}_{T} d z d \varphi_{R} d M_{h}^{2} d \xi d \bar{z} d y},
$$

where the unpolarized integrated cross section in eq. (2.50) is simply

$$
\frac{d \sigma\left(e^{+} e^{-} \rightarrow\left(h_{1} h_{2}\right)+\Lambda+X\right)}{d z d M_{h}^{2} d \bar{z} d y}=\langle 1\rangle .
$$

\subsection{The asymmetry induced by the Collins effect for unpolarized $\Lambda$}

For an unpolarized $\Lambda$, only the first and the last terms in eq. (2.41) contribute. In addition to the unpolarized term, the correlation of the transverse polarizations of the fragmenting quark and antiquark pair is described by the convolution of the Collins function $H_{1}^{\perp, \bar{a} \rightarrow \Lambda}$ for a $\Lambda$ with the two dihadron analogues $H_{1}^{\perp, a \rightarrow h_{1} h_{2}}, H_{1}^{\varangle, a \rightarrow h_{1} h_{2}}$ for the hadron pair $h_{1} h_{2}$. Thus, we can access this term by considering the following weighted average

$$
\begin{aligned}
& \left\langle\frac{q_{T}}{M_{\Lambda}} \cos \left(\varphi_{q}+\varphi_{R}\right)\right\rangle=\frac{3 \alpha_{e m}^{2}}{(2 \pi)^{2} Q^{2}} \frac{B(y)}{M_{\Lambda}^{2} M_{h}} \\
& \quad \times \sum_{a} e_{a}^{2} \int d \xi \int d \varphi_{R} \int d^{2} \boldsymbol{q}_{T} \int d^{2} \boldsymbol{k}_{T} \int d^{2} \overline{\boldsymbol{k}}_{T} \delta^{2}\left(\boldsymbol{k}_{T}+\overline{\boldsymbol{k}}_{T}-\boldsymbol{q}_{T}\right) q_{T} \cos \left(\varphi_{q}+\varphi_{R}\right) \\
& \times\left[\left(k_{T} \bar{k}_{T} \cos \left(\varphi_{k}+\varphi_{\bar{k}}\right) H_{1}^{\perp, a \rightarrow h_{1} h_{2}}+R_{T} \bar{k}_{T} \cos \left(\varphi_{R}+\varphi_{\bar{k}}\right) H_{1}^{\varangle, a \rightarrow h_{1} h_{2}}\right) H_{1}^{\perp, \bar{a} \rightarrow \Lambda}\right],
\end{aligned}
$$

where we can use the $\delta$ function to write

$$
\int d^{2} \boldsymbol{q}_{T} \delta^{2}\left(\boldsymbol{k}_{T}+\overline{\boldsymbol{k}}_{T}-\boldsymbol{q}_{T}\right) q_{T} \cos \left(\varphi_{q}+\varphi_{R}\right)=\left(k_{T} \cos \left(\varphi_{k}+\varphi_{R}\right)+\bar{k}_{T} \cos \left(\varphi_{\bar{k}}+\varphi_{R}\right)\right) .
$$

We further use the Fourier cosine decomposition for the DiFFs in eq. (2.38) to conclude

$$
\left\langle\frac{q_{T}}{M_{\Lambda}} \cos \left(\varphi_{q}+\varphi_{R}\right)\right\rangle=\frac{3 \alpha_{e m}^{2}}{(2 \pi)^{2} Q^{2}} B(y) \sum_{a} e_{a}^{2} H_{1}^{\varangle, a \rightarrow h_{1} h_{2}}\left(z, M_{h}^{2}\right) H_{1}^{\perp \bar{a},[1]}(\bar{z}),
$$

noting that

$$
\begin{aligned}
H_{1}^{\varangle, a \rightarrow h_{1} h_{2}} & \left(z, M_{h}^{2}\right) \\
& \equiv z^{2} \int d^{2} \boldsymbol{k}_{T} \int d \xi\left[\frac{\left|\boldsymbol{R}_{T}\right|}{M_{h}} H_{1}^{\varangle,[0]}\left(z, \xi,\left|\boldsymbol{k}_{T}\right|,\left|\boldsymbol{R}_{T}\right|\right)+\frac{\left|\boldsymbol{k}_{T}\right|}{M_{h}} H_{1}^{\perp,[1]}\left(z, \xi,\left|\boldsymbol{k}_{T}\right|,\left|\boldsymbol{R}_{T}\right|\right)\right],
\end{aligned}
$$


is the same integrated IFF that enters in SIDIS [17] and in $e^{+} e^{-}$two hadron pair [23] asymmetries, while

$$
H_{1}^{\perp \bar{a},[1]}(\bar{z}) \equiv \bar{z}^{2} \int d^{2} \overline{\boldsymbol{k}}_{T} \frac{\overline{\boldsymbol{k}}_{T}^{2}}{2 M_{\Lambda}^{2}} H_{1}^{\perp, \bar{a} \rightarrow \Lambda}\left(z, \overline{\boldsymbol{k}}_{T}^{2}\right)
$$

is the first moment of the Collins function.

The corresponding weighted azimuthal asymmetry then takes a simple form

$$
A^{\text {Coll }}=\frac{B(y)}{A(y)} \frac{\sum_{a} e_{a}^{2} H_{1}^{\varangle, a \rightarrow h_{1} h_{2}}\left(z, M_{h}^{2}\right) H_{1}^{\perp \bar{a},[1]}(\bar{z})}{\sum_{a} e_{a}^{2} D_{1}^{a \rightarrow h_{1} h_{2}}\left(z, M_{h}^{2}\right) \bar{D}_{1}^{\bar{a} \rightarrow \Lambda}(\bar{z})} .
$$

\subsection{The asymmetries for a longitudinally polarized $\Lambda$}

To devise the measurements involving the polarization of the produced $\Lambda$, it is important to underline that the cross section expression in eq. (2.41) is a conditional probability of the process for the given polarization $\boldsymbol{S}_{\Lambda}$. We have no a priory knowledge of this polarization, and it cannot be measured directly on an event-by-event basis. Thus, we need to extract the polarization $\boldsymbol{s}_{\Lambda}$ that $\Lambda$ acquires in the back-to-back fragmentation process, which can be measured in experiment by considering the average angular distributions of the final state particles. Employing the spin density matrix formalism, we can infer from general considerations [29, 41, 42], that the cross section of this process can be expressed as

$$
\frac{d \sigma}{d V}=\alpha+\boldsymbol{\beta} \cdot \boldsymbol{S}_{\Lambda} \sim 1+\boldsymbol{S}_{\Lambda} \cdot \boldsymbol{s}_{\Lambda}
$$

Thus, the acquired polarization vector is simply given by

$$
\boldsymbol{s}_{\Lambda}=\frac{\boldsymbol{\beta}}{\alpha},
$$

where $\alpha$ and $\boldsymbol{\beta}$ are only functions of the momentum of $\Lambda$ and the polarization of the fragmenting quark. The latter in our work is expressed using the correlations with the momenta of $h_{1}, h_{2}$, so that the acquired polarization $s_{\Lambda}$ only depends on the momenta of the observed particles. Further, it is clear from eq. (3.9), that the average of the acquired polarization over the kinematic variables is given by

$$
\left\langle s_{\Lambda}\right\rangle=\frac{\langle\boldsymbol{\beta}\rangle}{\langle\alpha\rangle},
$$

as the probability of the process itself is given by $\alpha$. The coefficients $\alpha$ and $\boldsymbol{\beta}$ can be easily read off directly from eq. (2.41). For example, $\alpha$ corresponds to the first and the last terms on the right hand side of eq. (2.41), while the longitudinal part $\beta_{L}$ of the coefficient $\boldsymbol{\beta}$ is given by the terms multiplied by $\lambda_{\Lambda}$. In section 4 we present an example of a measurement for the longitudinal polarization of $\Lambda^{0}$ baryon.

Let us consider the correlations for the longitudinally polarized $\Lambda$ that are manifest in two terms in eq. (2.41). The term that involves $G_{1}^{\perp, a \rightarrow h_{1} h_{2}}$ and $G_{1 L}^{\bar{a} \rightarrow \Lambda}$ describes the 
correlations between the longitudinal polarizations of the fragmenting quark and antiquark. We can access the helicity of $\Lambda$ by considering the weighted average

$$
\left\langle\beta_{L}\right\rangle_{G_{1}^{\perp} G_{1 L}}=\left\langle\frac{q_{T}}{M_{h}} \sin \left(\varphi_{q}-\varphi_{R}\right)\right\rangle=\frac{3 \alpha_{e m}^{2}}{(2 \pi)^{2} Q^{2}} A(y) \sum_{a} e_{a}^{2} G_{1}^{\perp, a \rightarrow h_{1} h_{2}}\left(z, M_{h}^{2}\right) G_{1 L}^{\bar{a} \rightarrow \Lambda}(\bar{z}),
$$

where

$$
\begin{aligned}
G_{1}^{\perp, a \rightarrow h_{1} h_{2}} & \left(z, M_{h}^{2}\right) \\
& \equiv z^{2} \int d^{2} \boldsymbol{k}_{T} \frac{\boldsymbol{k}_{T}^{2}}{2 M_{h}^{2}} \int d \xi \frac{R_{T}}{M_{h}}\left(G_{1}^{\perp,[0]}\left(z, \xi,\left|\boldsymbol{k}_{T}\right|,\left|\boldsymbol{R}_{T}\right|\right)-G_{1}^{\perp,[2]}\left(z, \xi,\left|\boldsymbol{k}_{T}\right|,\left|\boldsymbol{R}_{T}\right|\right)\right),
\end{aligned}
$$

is the same integrated helicity-dependent DiFF as in refs. [20, 23] and

$$
G_{1 L}^{\bar{a} \rightarrow \Lambda}(\bar{z}) \equiv \bar{z}^{2} \int d^{2} \overline{\boldsymbol{k}}_{T} G_{1 L}^{\bar{a} \rightarrow \Lambda}\left(\bar{z}, \overline{\boldsymbol{k}}_{T}^{2}\right),
$$

is the integrated helicity FF. Thus, the acquired longitudinal polarization $s_{L}$ of $\Lambda$ is

$$
\left\langle s_{L}\right\rangle^{\sin \left(\varphi_{q}-\varphi_{R}\right)}\left(z, M_{h}^{2}, \bar{z}, y\right)=\frac{\sum_{a} e_{a}^{2} G_{1}^{\perp, a \rightarrow h_{1} h_{2}}\left(z, M_{h}^{2}\right) G_{1 L}^{\bar{a} \rightarrow \Lambda}(\bar{z})}{\sum_{a} e_{a}^{2} D_{1}^{a \rightarrow h_{1} h_{2}}\left(z, M_{h}^{2}\right) \bar{D}_{1}^{\bar{a} \rightarrow \Lambda}(\bar{z})},
$$

A second contribution is acquired by the correlation of the transverse polarizations of the quark and the anti quark, which couples the two analogues of Collin DiFFs on one side with the "Kotzinian-Mulders" type FF. The relevant weighting for this term is

$$
\left\langle\beta_{L}\right\rangle_{H_{1}^{\varangle} H_{1 L}^{\perp}}=\left\langle\frac{q_{T}}{M_{\Lambda}} \sin \left(\varphi_{q}+\varphi_{R}\right)\right\rangle=\frac{3 \alpha_{e m}^{2}}{(2 \pi)^{2} Q^{2}} B(y) \sum_{a} e_{a}^{2} H_{1}^{\varangle, a \rightarrow h_{1} h_{2}}\left(z, M_{h}^{2}\right) H_{1 L}^{\perp \bar{a},[1]}(\bar{z}),
$$

where

$$
H_{1 L}^{\perp \bar{a},[1]}(\bar{z}) \equiv \bar{z}^{2} \int d^{2} \overline{\boldsymbol{k}}_{T} \frac{\overline{\boldsymbol{k}}_{T}^{2}}{2 M_{\Lambda}^{2}} H_{1 L}^{\perp, \bar{a} \rightarrow \Lambda}\left(z, \overline{\boldsymbol{k}}_{T}^{2}\right) .
$$

The corresponding acquired helicity is

$$
\left\langle s_{L}\right\rangle^{\sin \left(\varphi_{q}+\varphi_{R}\right)}\left(z, M_{h}^{2}, \bar{z}, y\right)=\frac{B(y)}{A(y)} \frac{\sum_{a} e_{a}^{2} H_{1}^{\varangle, a \rightarrow h_{1} h_{2}}\left(z, M_{h}^{2}\right) H_{1 L}^{\perp \bar{a},[1]}(\bar{z})}{\sum_{a} e_{a}^{2} D_{1}^{a \rightarrow h_{1} h_{2}}\left(z, M_{h}^{2}\right) \bar{D}_{1}^{\bar{a} \rightarrow \Lambda}(\bar{z})} .
$$

\subsection{The asymmetries for a transversely polarized $\Lambda$}

Finally, we extract the correlations for the transverse polarization of $\Lambda$. The are four relevant terms contributing to the transverse part of $\boldsymbol{\beta}_{T}$ in eq. (2.41). Here we can proceed by considering the $\hat{x}$ and $\hat{y}$ components of this vector, labelled $\beta_{x}$ and $\beta_{y}$, respectively.

The transverse polarization acquired by the "polarizing" FF, $D_{1 T}^{\perp}$ mixes with the contribution involving the transversity- and pretzelocity-like FFs of $\Lambda$.

$$
\begin{aligned}
\left\langle\beta_{x}\right\rangle^{\sin \left(\varphi_{q}\right)}= & \left\langle q_{T} \sin \left(\varphi_{q}\right)\right\rangle \\
= & \frac{3 \alpha_{e m}^{2}}{(2 \pi)^{2} Q^{2}} \sum_{a} e_{a}^{2}\left\{-A(y) M_{\Lambda} D_{1}^{a \rightarrow h_{1} h_{2}}\left(z, M_{h}^{2}\right) D_{1 T}^{\perp \bar{a},[1]}(\bar{z})\right. \\
& \left.\quad+B(y) M_{h} H_{1}^{\perp, a \rightarrow h_{1} h_{2}}\left(z, M_{h}^{2}\right) H_{1}^{\bar{a} \rightarrow \Lambda}(\bar{z})\right\},
\end{aligned}
$$




$$
\begin{aligned}
\left\langle\beta_{y}\right\rangle^{\cos \left(\varphi_{q}\right)}= & \left\langle q_{T} \cos \left(\varphi_{q}\right)\right\rangle \\
= & \frac{3 \alpha_{e m}^{2}}{(2 \pi)^{2} Q^{2}} \sum_{a} e_{a}^{2}\left\{A(y) M_{\Lambda} D_{1}^{a \rightarrow h_{1} h_{2}}\left(z, M_{h}^{2}\right) D_{1 T}^{\perp \bar{a},[1]}(\bar{z})\right. \\
& \left.\quad+B(y) M_{h} H_{1}^{\perp, a \rightarrow h_{1} h_{2}}\left(z, M_{h}^{2}\right) H_{1}^{\bar{a} \rightarrow \Lambda}(\bar{z})\right\},
\end{aligned}
$$

where

$$
\begin{aligned}
H_{1}^{\perp, a} & \rightarrow h_{1} h_{2}\left(z, M_{h}^{2}\right) \\
& \equiv z^{2} \int d^{2} \boldsymbol{k}_{T} \int d \xi\left[\frac{\left|\boldsymbol{k}_{T}\right|\left|\boldsymbol{R}_{T}\right|}{2 M_{h}^{2}} H_{1}^{\varangle,[1]}\left(z, \xi,\left|\boldsymbol{k}_{T}\right|,\left|\boldsymbol{R}_{T}\right|\right)+\frac{\left|\boldsymbol{k}_{T}\right|^{2}}{2 M_{h}^{2}} H_{1}^{\perp,[0]}\left(z, \xi,\left|\boldsymbol{k}_{T}\right|,\left|\boldsymbol{R}_{T}\right|\right)\right],
\end{aligned}
$$

and

$$
\begin{aligned}
D_{1 T}^{\perp \bar{a},[1]}(\bar{z}) & \equiv \bar{z}^{2} \int d^{2} \overline{\boldsymbol{k}}_{T} \frac{\overline{\boldsymbol{k}}_{T}^{2}}{2 M_{\Lambda}^{2}} D_{1 T}^{\perp \bar{a},[1]}\left(\bar{z}, \overline{\boldsymbol{k}}_{T}^{2}\right), \\
H_{1}^{\bar{a}}(\bar{z}) & \equiv \bar{z}^{2} \int d^{2} \overline{\boldsymbol{k}}_{T}\left(H_{1 T}^{\bar{a}}\left(\bar{z}, \overline{\boldsymbol{k}}_{T}^{2}\right)+\frac{\overline{\boldsymbol{k}}_{T}^{2}}{2 M_{\Lambda}^{2}} H_{1 T}^{\perp \bar{a}}\left(\bar{z}, \overline{\boldsymbol{k}}_{T}^{2}\right)\right) .
\end{aligned}
$$

This is analogous to the associated $\Lambda$ production asymmetry proposed in ref. [19] and the additional $q_{T}$ weighting in our case allows us to disentangle the convolution of $\Lambda$ FFs and DiFFs into "collinear" products of the corresponding moments. Here, the contributions involving the chiral-odd FFs, ignored in [19], enter with different relative sign to those containing the "polarizing" FF. Thus, we can form linear combinations of the two terms to access the contributions from the individual structure functions

$$
\begin{gathered}
\left\langle\beta_{y}\right\rangle^{\cos \left(\varphi_{q}\right)}-\left\langle\beta_{x}\right\rangle^{\sin \left(\varphi_{q}\right)}=M_{\Lambda} \frac{3 \alpha_{e m}^{2}}{2 \pi^{2} Q^{2}} A(y) \sum_{a} e_{a}^{2} D_{1}^{a \rightarrow h_{1} h_{2}}\left(z, M_{h}^{2}\right) D_{1 T}^{\perp \bar{a},[1]}(\bar{z}), \\
\left\langle\beta_{y}\right\rangle^{\cos \left(\varphi_{q}\right)}+\left\langle\beta_{x}\right\rangle^{\sin \left(\varphi_{q}\right)}=M_{h} \frac{3 \alpha_{e m}^{2}}{2 \pi^{2} Q^{2}} B(y) \sum_{a} e_{a}^{2} H_{1}^{\perp, a \rightarrow h_{1} h_{2}}\left(z, M_{h}^{2}\right) H_{1}^{\bar{a} \rightarrow \Lambda}(\bar{z}),
\end{gathered}
$$

and the corresponding polarizations

$$
\begin{aligned}
& \frac{\left\langle s_{y}\right\rangle^{\cos \left(\varphi_{q}\right)}\left(z, M_{h}^{2}, \bar{z}, y\right)-\left\langle s_{x}\right\rangle^{\sin \left(\varphi_{q}\right)}\left(z, M_{h}^{2}, \bar{z}, y\right)}{M_{\Lambda}} \\
& =2 \frac{\sum_{a} e_{a}^{2} D_{1}^{a \rightarrow h_{1} h_{2}}\left(z, M_{h}^{2}\right) D_{1 T}^{\perp \bar{a},[1]}(\bar{z})}{\sum_{a} e_{a}^{2} D_{1}^{a \rightarrow h_{1} h_{2}}\left(z, M_{h}^{2}\right) \bar{D}_{1}^{\bar{a} \rightarrow \Lambda}(\bar{z})}, \\
& \frac{\left\langle s_{y}\right\rangle^{\cos \left(\varphi_{q}\right)}\left(z, M_{h}^{2}, \bar{z}, y\right)+\left\langle s_{x}\right)^{\sin \left(\varphi_{q}\right)}\left(z, M_{h}^{2}, \bar{z}, y\right)}{M_{h}} \\
& =\frac{2 B(y)}{A(y)} \frac{\sum_{a} e_{a}^{2} H_{1}^{\perp, a \rightarrow h_{1} h_{2}}\left(z, M_{h}^{2}\right) H_{1}^{\bar{a} \rightarrow \Lambda}(\bar{z})}{\sum_{a} e_{a}^{2} D_{1}^{a \rightarrow h_{1} h_{2}}\left(z, M_{h}^{2}\right) \bar{D}_{1}^{\bar{a} \rightarrow \Lambda}(\bar{z})} .
\end{aligned}
$$

It is important to stress, that here we are operating with the measured polarizations along the $\hat{x}$ and $\hat{y}$.

The contribution from the term involving $G_{1}^{\perp, a \rightarrow h_{1} h_{2}}$ and another "worm-gear" type $\mathrm{FF}, G_{1 T}^{\bar{a}}$, which describes the longitudinal quark antiquark polarization correlations, also 
admixes with the contributions from several other terms when calculating the weighted moments, for example with a weight $\left\langle q_{T}^{2} \sin \left(\varphi_{q}-\varphi_{R}\right) \cos \left(\varphi_{q}\right)\right\rangle$. Here, we omit the resulting long and convoluted expression (even by the standards of this manuscript) for brevity.

The last contribution to the transverse polarization can be obtained using weights that only involve the azimuthal angle $\varphi_{R}$ and no additional factors of $q_{T}$

$$
\left\langle\boldsymbol{\beta}_{x}\right\rangle_{H_{1}^{\varangle} H_{1}}^{\sin \left(\varphi_{R}\right)}=\left\langle\boldsymbol{\beta}_{y}\right\rangle_{H_{1}^{\varangle} H_{1}}^{\cos \left(\varphi_{R}\right)}=\frac{3 \alpha_{e m}^{2}}{8 \pi^{2} Q^{2}} B(y) \sum_{a} e_{a}^{2} H_{1}^{\varangle, a \rightarrow h_{1} h_{2}}\left(z, M_{h}^{2}\right) H_{1}^{\bar{a} \rightarrow \Lambda}(\bar{z}) .
$$

The corresponding acquired transverse polarization is

$$
\begin{aligned}
\left\langle\boldsymbol{s}_{T}\right\rangle_{x}^{\sin \left(\varphi_{R}\right)}\left(z, M_{h}^{2}, \bar{z}, y\right) & =\left\langle\boldsymbol{s}_{T}\right\rangle_{y}^{\cos \left(\varphi_{R}\right)}\left(z, M_{h}^{2}, \bar{z}, y\right) \\
& =\frac{1}{2} \frac{B(y)}{A(y)} \frac{\sum_{a} e_{a}^{2} H_{1}^{\varangle, a \rightarrow h_{1} h_{2}}\left(z, M_{h}^{2}\right) H_{1}^{\bar{a}}(\bar{z})}{\sum_{a} e_{a}^{2} D_{1}^{a \rightarrow h_{1} h_{2}}\left(z, M_{h}^{2}\right) \bar{D}_{1}^{\bar{a} \rightarrow \Lambda}(\bar{z})} .
\end{aligned}
$$

This is similar to the IFF asymmetry in SIDIS, where the IFF is multiplied by the integrated transversity PDF. Here, similar to the integrated transversity PDF, the polarized FF $H_{1}$ is a combination of the TMD "transversity" FF $H_{1 T}$ and TMD "pretzelocity" $H_{1 T}^{\perp}$, see eq. (3.23).

\section{Treatment of the polarized $\Lambda^{0}$}

In this section we will elaborate the treatment of the measurements involving polarized $\Lambda^{0}$ production, where either the longitudinal or the transverse polarization is leveraged to access the relevant structure functions in the cross section. The determination of the final state polarization is possible for this hyperon, as it undergoes "self-analyzing" weak decays into a baryon-meson pair. The two hadronic decay channels into $p+\pi^{-}$and $n+\pi^{0}$ have relative branching ratios of $64 \%$ and $36 \%$, respectively. An important aspect of such decays is the correlation between the polarization vector of the decaying hyperon and the produced hadron's momentum. In particular, taking the $p+\pi^{-}$channel as an example, the decay rate is described as

$$
\frac{d N}{N d \cos \theta} \sim 1+\alpha_{\Lambda} S_{\Lambda} \cos (\theta)
$$

where $S_{\Lambda}$ is the modulus of the polarization of the $\Lambda^{0}$, and $\theta$ is the angle between the decay proton momentum and the $\Lambda^{0}$ polarization vector in its rest frame. For the longitudinal polarization of the $\Lambda^{0}$ this angle is calculated along the direction of the $\hat{z}$ axis defined in section I, figure 1 , as we can simply boost along $\hat{z}$ to the $\Lambda^{0}$ rest frame. The decay parameter $\alpha_{\Lambda}$ has been measured in a number of experiments in the 60's and 70's [43], with the Particle Data Group average value of $\alpha_{\Lambda}=0.642$ [44], which describes the admixture of $s-$ and $p-$ partial waves of the $p, \pi^{-}$system [45].

The idea of using $\Lambda^{0}$ 's as a quark longitudinal spin polarimeter in SIDIS and $e^{+} e^{-} \rightarrow$ $\Lambda^{0}+X$ annihilation reactions was originally proposed in refs. [46-48] and for a quark transverse spin polarimeter in SIDIS in ref. [49]. Since then a number of experimental and 
phenomenological studies of hyperon polarization in these reactions have been performed, see for examples refs. [50-56]. It is worth mentioning that substantial longitudinal polarization of $\Lambda^{0}\left(\lambda_{\Lambda}=-0.320\right.$ for $\left.z>0.3\right)$ was observed by ALEPH ref. [50], indicating the large analyzing power of this measurement. Similarly, the BELLE collaboration has recently measured a transverse polarization of the $\Lambda^{0}$ of order 0.1 via the so-called "polarizing" fragmentation function ref. $[57,58]$, observing significant analyzing power for the transverse polarization as well.

The important aspect of our proposed measurements is that the polarization of the $\Lambda^{0}$ couples with the azimuthal angles of the dihadron pair on the other side. On the other hand, the hyperon itself is not directly detected in the experiment, but rather is seen through its decay products. In such a decay, the polarization of the $\Lambda^{0}$ induces modulations of the angular distributions of the decay products. For example, for the helicity-dependent modulations involving the helicity $\lambda_{\Lambda}$, the polar angle of the decay product $p$ will be modulated by $1+\lambda_{\Lambda} \cos \left(\theta_{p}\right)$, where $\theta_{p}$ is the polar angle of the proton in the rest frame of $\Lambda^{0}$.

Thus, we need to consider the full observed final state in our process $e^{+} e^{-} \rightarrow\left(h_{1} h_{2}\right)+$ $\Lambda^{0}+X \rightarrow\left(h_{1} h_{2}\right)+\left(p+\pi^{-}\right)+X$. For extraction of the asymmetry in eq. (3.15), we should consider a weighted integral of the complete final state

$$
\left\langle\cos \left(\theta_{p}\right) \frac{q_{T}}{M_{h}} \sin \left(\varphi_{q}-\varphi_{R}\right)\right\rangle \sim \alpha_{\Lambda} G_{1}^{\perp, a \rightarrow h_{1} h_{2}} G_{1 L}^{\bar{a} \rightarrow \Lambda},
$$

where the measurement of the weighted asymmetries with the detected final state hadrons now involves also the integration over the phase space of the proton. Similar expressions can also be derived for the components of the transverse polarization of $\Lambda^{0}$.

Such measurements should be possible with the much improved apparatus of the BELLE II Collaboration in the near future.

\section{Conclusions}

The study of the quark hadronization in $e^{+} e^{-}$experiments is a crucial part of the international efforts to map the 3-dimensional structure of the nucleon and to understand the spin-orbit correlations in the strong interactions. The measurements of the Collins effect and the two-hadron interference effect at BELLE [2-5] and BaBar [6] have been used to extract the nucleon transversity PDF.

The theoretical underpinning for such measurements of the Collins effect in two backto-back hadron production was given by Boer and collaborators in refs. [24, 25, 28], where the relevant asymmetry involves convolution of two Collins FFs for the produced hadrons on each side. A measurement with a polarized hadron on one side and an unpolarized one on the other side was proposed in ref. [19] to test the universality of the T-odd "polarizing" FF entering the corresponding cross section. Recently, the work by [59] developed an approach for calculations of the polarized back-to-back hadron pair production in $e^{+} e^{-}$ in an arbitrary frame and to arbitrary precision. The measurement of the spin-dependent DiFF was first proposed in ref. [22] and recently revised and corrected in ref. [23]. Here, 
the cross section for producing two back-to-back hadron pairs includes convolutions of two DiFFs, one for each pair involved. Note, that the unpolarized FFs can be measured in single inclusive hadron production, while the unpolarized DiFFs are measured by detecting two inclusive hadrons in the same jet [5].

In this work, we proposed a new inclusive measurement, where an unpolarized hadron pair is detected back-to-back with a single hadron, that may or may not be polarized. We derived the expression for the corresponding cross section in eq. (2.41), which involves convolutions of the DiFFs for the hadron pair with the FFs for the hadron on the other side. A number of new exciting measurements were then discussed. For example, the measurement of the unpolarized cross section will enable us to determine the flavor dependence of the DiFF using our knowledge of the ordinary unpolarized FFs, which is not possible with the other $e^{+} e^{-}$measurements. Moreover, we can measure a product of the Collins function of a meson with the IFF of the hadron pair eq. (3.8), that would provide additional information to constrain both functions. This is crucial in improving our knowledge of the transversity PDF from SIDIS measurements involving one and two hadron inclusive final states.

The measurement of the hadron polarization dependent asymmetries gives access to a wide variety of combinations of polarized spin $1 / 2$ baryon FFs and polarized DiFFs, as discussed in section 3. Here we have eight TMD FFs for the baryon convoluted with four DiFFs for the unpolarized hadron pair, whereas in the case of two back-to-back dihadron pairs there are only the four DiFFs involved from each side [23]. Nonetheless, further developing the weighted asymmetry method we used in accessing the helicity-dependent DiFFs in ref. [20], we were able to access the individual combination of TMD FFs with DiFFs, disentangling their transverse momentum convolutions.

In section 3.2 we discussed a critical point for the measurements involving a polarized final state hadron. This polarization is acquired during the hadronization process, in correlation with the momenta of the observed final state particles. Using the spin density matrix formalism, we expressed the polarization vector of $\Lambda$ by utilizing the eq. (2.41). Then the measurements of different components of this polarization when analyzed using a particular weighted azimuthal modulations allows us to access specific combinations of FFs with DiFFs. For example, the weighted average in eq. (3.15) extracts a product of the helicity-dependent DiFF $G_{1}^{\perp}$ with the "collinear" helicity FF $G_{1 L}$ when measuring the longitudinal polarization of the $\Lambda$. Such a measurement will complement the previously proposed measurements of $G_{1}^{\perp}$ in $e^{+} e^{-}$annihilation and SIDIS [20,60], aimed at testing the universality of $G_{1}^{\perp}$. The transverse polarization-dependent modulation in eq. (3.29) yields a product of the collinear IFF with the collinear "transversity" FF. The last one is strikingly similar to SIDIS, where a similar modulation yields a product of the IFF with the transversity PDF [17].

Another interesting example is the measurement of the transverse polarization components of the hadron for the weighted azimuthal modulation in eq. (3.19), involving the components of the transverse momentum $\boldsymbol{q}_{T}$, which yields an admixture of two terms. One is the product of the unpolarized DiFF with the T-odd "polarizing" FF of $\Lambda$, while the second is the product of the Collins-like DiFF with transversity-like FF of $\Lambda$. This is an analogue of the associated production measurement proposed in ref. [19], where the contri- 
butions of the chiral-odd functions (such as Collins function and the transversity-like FF) were neglected. Note, that only the convolution of the chiral-odd unpolarized DiFF with the chiral-odd "polarizing" FF contribute to the perpendicular components of polarization to $\boldsymbol{P}_{h \perp}$, which was considered in Ref. [19]. Our results demonstrate that we can separate the chiral-odd and chiral-even contributions by forming linear combinations of the $x$ - and $y$-components of the transverse polarization.

The measurement of the baryon polarization was discussed in section 4 . Such measurements for the $\Lambda^{0}$ hyperon through the "self-analyzing" weak decays have been long employed as polarimeters. Here, we discussed the relevant weighted asymmetries for the full detected final state particles to measure the relevant structure functions within the "narrow width approximation" for the treatment if the intermediate $\Lambda^{0}$ baryon, as described for the longitudinal polarization case in eq. (4.2).

This new process completes the set of all possible inclusive measurements with up to two produced hadrons on each side. It presents an exciting new opportunity for exploring the hadronization process using data already collected by the BELLE experiment, as well as that to be taken in the upcoming BELLE II experiment. A combined global analysis of these measurements along with the other inclusive hadron production reactions in electronpositron annihilation will allow us to acquire a good understanding of both single hadron and dihadron fragmentations.

\section{Acknowledgments}

We would like to thank Daniël Boer(Groningen) for a useful discussion. The work of H.H.M. and A.W.T. was supported by the Australian Research Council through the ARC Centre of Excellence for Particle Physics at the Terascale (CE110001104) and by the ARC Discovery Project No. DP151103101, as well as by the University of Adelaide. A.K. was supported by A.I. Alikhanyan National Science Laboratory (YerPhI) Foundation, Yerevan, Armenia.

Open Access. This article is distributed under the terms of the Creative Commons Attribution License (CC-BY 4.0), which permits any use, distribution and reproduction in any medium, provided the original author(s) and source are credited.

\section{References}

[1] A. Metz and A. Vossen, Parton fragmentation functions, Prog. Part. Nucl. Phys. 91 (2016) 136 [arXiv: 1607.02521] [INSPIRE].

[2] Belle collaboration, K. Abe et al., Measurement of azimuthal asymmetries in inclusive production of hadron pairs in $e^{+} e^{-}$annihilation at Belle, Phys. Rev. Lett. 96 (2006) 232002 [hep-ex/0507063] [INSPIRE].

[3] BeLle collaboration, R. Seidl et al., Measurement of azimuthal asymmetries in inclusive production of hadron pairs in $e^{+} e^{-}$annihilation at $\sqrt{s}=10.58 \mathrm{GeV}$, Phys. Rev. D 78 (2008) 032011 [Erratum ibid. D 86 (2012) 039905] [arXiv:0805.2975] [INSPIRE]. 
[4] Belle collaboration, A. Vossen et al., Observation of transverse polarization asymmetries of charged pion pairs in $e^{+} e^{-}$annihilation near $\sqrt{s}=10.58$ GeV, Phys. Rev. Lett. 107 (2011) 072004 [arXiv: 1104.2425] [INSPIRE].

[5] Belle collaboration, R. Seidl et al., Invariant-mass and fractional-energy dependence of inclusive production of di-hadrons in $e^{+} e^{-}$annihilation at $\sqrt{s}=10.58$ GeV, Phys. Rev. D 96 (2017) 032005 [arXiv: 1706.08348] [INSPIRE].

[6] BABAR collaboration, J.P. Lees et al., Measurement of collins asymmetries in inclusive production of charged pion pairs in $e^{+} e^{-}$annihilation at BABAR, Phys. Rev. D 90 (2014) 052003 [arXiv: 1309.5278] [INSPIRE].

[7] Z.-B. Kang, A. Prokudin, P. Sun and F. Yuan, Extraction of quark transversity distribution and collins fragmentation functions with QCD evolution, Phys. Rev. D 93 (2016) 014009 [arXiv: 1505.05589] [INSPIRE].

[8] M. Anselmino et al., Collins functions for pions from SIDIS and new $e^{+} e^{-}$data: a first glance at their transverse momentum dependence, Phys. Rev. D 92 (2015) 114023 [arXiv: 1510.05389] [INSPIRE].

[9] A. Bacchetta, A. Courtoy and M. Radici, First glances at the transversity parton distribution through dihadron fragmentation functions, Phys. Rev. Lett. 107 (2011) 012001 [arXiv:1104.3855] [INSPIRE].

[10] S. Pisano and M. Radici, Di-hadron fragmentation and mapping of the nucleon structure, Eur. Phys. J. A 52 (2016) 155 [arXiv:1511.03220] [InSPIRE].

[11] HERMES collaboration, A. Airapetian et al., Single-spin asymmetries in semi-inclusive deep-inelastic scattering on a transversely polarized hydrogen target, Phys. Rev. Lett. 94 (2005) 012002 [hep-ex/0408013] [INSPIRE].

[12] HERMES collaboration, A. Airapetian et al., Evidence for a transverse single-spin asymmetry in leptoproduction of $\pi^{+} \pi^{-}$pairs, JHEP 06 (2008) 017 [arXiv:0803.2367] [INSPIRE].

[13] COMPASS collaboration, C. Adolph et al., Transverse spin effects in hadron-pair production from semi-inclusive deep inelastic scattering, Phys. Lett. B 713 (2012) 10 [arXiv: 1202.6150] [INSPIRE].

[14] COMPASS collaboration, C. Adolph et al., A high-statistics measurement of transverse spin effects in dihadron production from muon-proton semi-inclusive deep-inelastic scattering, Phys. Lett. B 736 (2014) 124 [arXiv:1401.7873] [INSPIRE].

[15] S. Meissner and A. Metz, Partonic pole matrix elements for fragmentation, Phys. Rev. Lett. 102 (2009) 172003 [arXiv:0812.3783] [INSPIRE].

[16] L.P. Gamberg, A. Mukherjee and P.J. Mulders, A model independent analysis of gluonic pole matrix elements and universality of TMD fragmentation functions, Phys. Rev. D 83 (2011) 071503 [arXiv: 1010.4556] [INSPIRE].

[17] A. Bacchetta and M. Radici, Two hadron semiinclusive production including subleading twist, Phys. Rev. D 69 (2004) 074026 [hep-ph/0311173] [INSPIRE].

[18] J.C. Collins, Leading twist single transverse-spin asymmetries: Drell-Yan and deep inelastic scattering, Phys. Lett. B 536 (2002) 43 [hep-ph/0204004] [INSPIRE].

[19] D. Boer, Z.-B. Kang, W. Vogelsang and F. Yuan, Test of the universality of naive-time-reversal-odd fragmentation functions, Phys. Rev. Lett. 105 (2010) 202001 [arXiv: 1008.3543] [INSPIRE]. 
[20] H.H. Matevosyan, A. Kotzinian and A.W. Thomas, Accessing quark helicity through dihadron studies, Phys. Rev. Lett. 120 (2018) 252001 [arXiv:1712.06384] [INSPIRE].

[21] A. Bacchetta and M. Radici, Partial wave analysis of two hadron fragmentation functions, Phys. Rev. D 67 (2003) 094002 [hep-ph/0212300] [InSPIRE].

[22] D. Boer, R. Jakob and M. Radici, Interference fragmentation functions in electron positron annihilation, Phys. Rev. D 67 (2003) 094003 [Erratum ibid. D 98 (2018) 039902] [hep-ph/0302232] [INSPIRE].

[23] H.H. Matevosyan et al., Semi-inclusive production of two back-to-back hadron pairs in $e^{+} e^{-}$ annihilation revisited, Phys. Rev. D 97 (2018) 074019 [arXiv: 1802.01578] [InSPIRE].

[24] D. Boer, R. Jakob and P.J. Mulders, Asymmetries in polarized hadron production in $e^{+} e^{-}$ annihilation up to order $1 / Q$, Nucl. Phys. B 504 (1997) 345 [hep-ph/9702281] [InSPIRE].

[25] D. Boer, R. Jakob and P.J. Mulders, Leading asymmetries in two hadron production in $e^{+} e^{-}$ annihilation at the $Z$ pole, Phys. Lett. B 424 (1998) 143 [hep-ph/9711488] [INSPIRE].

[26] A. Bianconi, S. Boffi, R. Jakob and M. Radici, Two hadron interference fragmentation functions. Part 1. General framework, Phys. Rev. D 62 (2000) 034008 [hep-ph/9907475] [INSPIRE].

[27] M. Radici, R. Jakob and A. Bianconi, Accessing transversity with interference fragmentation functions, Phys. Rev. D 65 (2002) 074031 [hep-ph/0110252] [INSPIRE].

[28] D. Boer, Angular dependences in inclusive two-hadron production at BELLE, Nucl. Phys. B 806 (2009) 23 [arXiv:0804.2408] [INSPIRE].

[29] P.J. Mulders and R.D. Tangerman, The complete tree level result up to order $1 / Q$ for polarized deep inelastic leptoproduction, Nucl. Phys. B 461 (1996) 197 [Erratum ibid. B 484 (1997) 538] [hep-ph/9510301] [INSPIRE].

[30] Belle collaboration, R. Seidl et al., Inclusive cross sections for pairs of identified light charged hadrons and for single protons in $e^{+} e^{-}$at $\sqrt{s}=10.58$ GeV, Phys. Rev. D 92 (2015) 092007 [arXiv: 1509.00563] [INSPIRE].

[31] D. de Florian et al., Parton-to-pion fragmentation reloaded, Phys. Rev. D 91 (2015) 014035 [arXiv: 1410.6027] [INSPIRE].

[32] M. Hirai, H. Kawamura, S. Kumano and K. Saito, Impacts of B-factory measurements on determination of fragmentation functions from electron-positron annihilation data, PTEP 2016 (2016) 113B04 [arXiv: 1608.04067] [INSPIRE].

[33] N. Sato et al., First Monte-Carlo analysis of fragmentation functions from single-inclusive $e^{+} e^{-}$annihilation, Phys. Rev. D 94 (2016) 114004 [arXiv: 1609.00899] [INSPIRE].

[34] J.J. Ethier, N. Sato and W. Melnitchouk, First simultaneous extraction of spin-dependent parton distributions and fragmentation functions from a global QCD analysis, Phys. Rev. Lett. 119 (2017) 132001 [arXiv: 1705.05889] [INSPIRE].

[35] NNPDF collaboration, V. Bertone et al., A determination of the fragmentation functions of pions, kaons and protons with faithful uncertainties, Eur. Phys. J. C 77 (2017) 516 [arXiv: 1706. 07049] [INSPIRE].

[36] T. Ito et al., The NJL-jet model for quark fragmentation functions, Phys. Rev. D 80 (2009) 074008 [arXiv: 0906 . 5362] [INSPIRE].

[37] H.H. Matevosyan, A.W. Thomas and W. Bentz, Analyzing unfavored fragmentation functions using NJL-jet model, AIP Conf. Proc. 1374 (2011) 387 [INSPIRE]. 
[38] H.H. Matevosyan, A.W. Thomas and W. Bentz, Monte Carlo simulations of hadronic fragmentation functions using NJL-jet model, Phys. Rev. D 83 (2011) 114010 [Erratum ibid. D 86 (2012) 059904] [arXiv:1103.3085] [INSPIRE].

[39] H.H. Matevosyan, A.W. Thomas and W. Bentz, Dihadron fragmentation functions within the NJL-jet model, Phys. Rev. D 88 (2013) 094022 [arXiv:1310.1917] [InSPIRE].

[40] D. Boer and P.J. Mulders, Time reversal odd distribution functions in leptoproduction, Phys. Rev. D 57 (1998) 5780 [hep-ph/9711485] [INSPIRE].

[41] V.B. Berestetskii, E.M. Lifshitz and L.P. Pitaevskii, Quantum electrodynamics, Course of Theoretical Physics volume 4, Pergamon Press, Oxford U.K. (1982).

[42] H.H. Matevosyan, A. Kotzinian and A.W. Thomas, Monte Carlo implementation of polarized hadronization, Phys. Rev. D 95 (2017) 014021 [arXiv: 1610.05624] [INSPIRE].

[43] J.W. Cronin and O.E. Overseth, Measurement of the decay parameters of the $\Lambda^{0}$ particle, Phys. Rev. 129 (1963) 1795 [INSPIRE].

[44] Particle Data Group collaboration, J. Beringer et al., Review of particle physics, Phys. Rev. D 86 (2012) 010001 [INSPIRE].

[45] T.D. Lee and C.-N. Yang, General partial wave analysis of the decay of a hyperon of spin 1/2, Phys. Rev. 108 (1957) 1645 [InSPIRE].

[46] I.I.Y. Bigi, Transfer of polarization from the initial to the final state in deep inelastic lepton scattering, Nuovo Cim. A 41 (1977) 43 [inSPIRE].

[47] I.I.Y. Bigi, Some quantitative estimates about final state polarization in deep inelastic lepton-nucleon scattering, Nuovo Cim. A 41 (1977) 581 [INSPIRE].

[48] J.E. Augustin and F.M. Renard, How to measure quark helicities in $e^{+} e^{-} \rightarrow$ hadrons, Nucl. Phys. B 162 (1980) 341 [inSPIRE].

[49] F. Baldracchini, N.S. Craigie, V. Roberto and M. Socolovsky, A survey of polarization asymmetries predicted by QCD, Fortsch. Phys. 30 (1981) 505 [InSPIRE].

[50] ALEPH collaboration, D. Buskulic et al., Measurement of $\Lambda$ polarization from $Z$ decays, Phys. Lett. B 374 (1996) 319 [inSPIRE].

[51] NOMAD collaboration, P. Astier et al., Measurement of the Lambda polarization in $\nu_{\mu}$ charged current interactions in the NOMAD experiment, Nucl. Phys. B 588 (2000) 3 [INSPIRE].

[52] COMPASS collaboration, M. Alekseev et al., Measurement of the longitudinal spin transfer to $\Lambda$ and anti- $\Lambda$ hyperons in polarised muon DIS, Eur. Phys. J. C 64 (2009) 171 [arXiv:0907.0388] [INSPIRE].

[53] J.R. Ellis, D. Kharzeev and A. Kotzinian, The proton spin puzzle and $\Lambda$ polarization in deep inelastic scattering, Z. Phys. C 69 (1996) 467 [hep-ph/9506280] [INSPIRE].

[54] A. Kotzinian, A. Bravar and D. von Harrach, $\Lambda$ and anti- $\Lambda$ polarization in lepton induced processes, Eur. Phys. J. C 2 (1998) 329 [hep-ph/9701384] [INSPIRE].

[55] J.R. Ellis, A. Kotzinian and D.V. Naumov, Intrinsic polarized strangeness and $\Lambda^{0}$ polarization in deep inelastic production, Eur. Phys. J. C 25 (2002) 603 [hep-ph/0204206] [INSPIRE].

[56] J.R. Ellis, A. Kotzinian, D. Naumov and M. Sapozhnikov, Longitudinal polarization of $\Lambda$ and anti- $\Lambda$ hyperons in lepton-nucleon deep-inelastic scattering, Eur. Phys. J. C 52 (2007) 283 [hep-ph/0702222] [INSPIRE]. 
[57] Belle collaboration, A. Abdesselam et al., Observation of transverse $\Lambda / \bar{\Lambda}$ hyperon polarization in $e^{+} e^{-}$annihilation at Belle, arXiv:1611.06648 [INSPIRE].

[58] BeLle collaboration, Y. Guan et al., Observation of transverse $\Lambda / \bar{\Lambda}$ hyperon polarization in $e^{+} e^{-}$annihilation at Belle, arXiv:1808.05000.

[59] D. Pitonyak, M. Schlegel and A. Metz, Polarized hadron pair production from electron-positron annihilation, Phys. Rev. D 89 (2014) 054032 [arXiv:1310.6240] [INSPIRE].

[60] H.H. Matevosyan, Accessing quark helicity in $e^{+} e^{-}$and SIDIS via dihadron correlations, arXiv: 1807.11485 [INSPIRE].

[61] H.H. Matevosyan, A. Kotzinian and A.W. Thomas, Studies of azimuthal modulations in two hadron fragmentation of a transversely polarised quark, Phys. Lett. B 731 (2014) 208 [arXiv:1312.4556] [INSPIRE]. 